\title{
Generalized Lindley-Quasi Xgamma distribution
}

\author{
S. A. WANI AND S. SHAFI
}

\begin{abstract}
We obtained a new generalization of Lindley-Quasi Xgamma distribution by adding weight parameter to it through weighting technique and have shown the flexibility of proposed model. Expression for reliability measures, order statistics, Bonferroni curves \& indices, Renyi entropy along with some other important properties are derived. Maximum likelihood estimation method is put to use for estimation of unknown parameters of proposed model. Simulation study for checking the performance of maximum likelihood estimates and for model comparison is carried out. Proposed model and its related models are fitted to real life data sets and goodness of fit measure Kolmogorov statistic \& p-value, loss of information criteria's AIC, BIC, AICC \& HQIC are computed through R software to check the applicability of proposed model in real life. The significance of weight parameter is also tested by using likelihood ratio test for both randomly generated data as well as real life data.
\end{abstract}

Mathematics Subject Classification 2010: 60E05, 62H10

Keywords: Weighting technique, Structural properties, Simulation study, Weight parameter, Bonferroni curves \& indices, Renyi entropy, Maximum likelihood estimation, Application.

\section{INTRODUCTION}

Probability models are and have been generalized for providing more flexibility in terms of hazard rate, reliability, prediction and moments. Because of presence of extra parameter generalized probability models find greater applicability in real life. Probability models can be generalized by using various methods. One of the method employed for generalizing probability models by adding weight parameter is weighting technique. Weighted models are mostly used in those situations where observations are recorded with unequal probabilities which generally happens in encountered sampling. Weighted models find applications in many fields of real life like forestry, medical sciences, socioeconomic surveys etc. Warren [1] applied the size biased distributions in connection with sampling wood cells. Hassan, Dar and Para [2] introduced a new generalization of Ishita distribution and obtained vital mathematical properties of the distribution along with applications of the proposed model. Hassan, Wani and Para [3] formulated three parameter Quasi Lindley 
distribution by using weighting technique and obtained various properties of that model. Das and Roy [4] studied the length biased weighted generalized Rayleigh distribution with properties and applications. Patil and Rao [5] introduced weighted distributions and size biased sampling with applications to wild life populations and human families and obtained its properties. Hassan, Wani and Shafi [6] introduced Poisson Pranav distribution and obtained its various mathematical properties along with obtaining applications of the proposed model. Rezaeia, Nadarajah and Tahghighnia [7] worked on a new three parameter life time distribution and studied its properties \& applications. Hassan, Wani, Bilal and Akhtar [8] introduced weighted Quasi Xgamma distribution and studied its properties and applications.

Hassan, Wani, Shafi and Sheikh [9] introduced Lindley-Quasi Xgamma Distribution (LQXD). With p.d.f, c.d.f and $c^{\text {th }}$ moment about origin $E\left(x^{c}\right)$ given below in (1.1), (1.2) and (1.3) respectively

$$
\begin{aligned}
& f(x, \alpha, \theta)=\frac{\theta \mathrm{e}^{-\theta \mathrm{x}}}{(\alpha+\theta)^{2}}\left\{(\alpha+\theta)\left(\alpha+\frac{\mathrm{x}^{2} \theta^{2}}{2}\right)+\theta(\theta-1)(1+\alpha x)\right\} \quad x>0, \theta>0, \alpha \geq 0 \\
& F(X)=\frac{1}{2(\theta+\alpha)^{2}}\left[\begin{array}{l}
\left.(\alpha+\theta)\left\{2 \alpha+2-\left(2 \alpha+x^{2} \theta^{2}+2 \theta x+2\right) e^{-\theta x}\right\}\right] \\
+2(\theta-1)\left\{\theta+\alpha-(\theta+\alpha \theta x+\alpha) e^{-\theta x}\right\}
\end{array}\right] \quad x>0, \theta>0, \alpha \geq 0 \\
& E\left(x^{c}\right)=\frac{c !\left\{(\theta+\alpha)\left(\alpha+\frac{(c+1)(c+2)}{2}\right)+(\theta-1)(\theta+\alpha(c+1))\right\}}{\theta^{c}(\theta+\alpha)^{2}}
\end{aligned}
$$

The important statistical properties along with application in real life were studied for the model given in (1.1).

In this paper we have obtained weighted version of Lindley-Quasi Xgamma (LQXD) distribution with p.d.f given in (1.1). 


\section{WEIGHTED LINDLEY-QUASI XGAMMA DISTRIBUTION}

Consider $\mathrm{X}$ to be a non-negative random variable following Lindley Quasi Xgamma distribution with p.d.f $f(x)$. Suppose $W(x)$ is the non-negative weight function, then the probability density function of the weighted Lindley Quasi Xgamma distribution (WLQXD) is

$f_{w}(x)=\frac{W(x) f(x)}{E(w(x))}, \quad x>0$

Where $w(x)=x^{c}$ is a non-negative weight function and $f(x), E\left(x^{c}\right)$ are given in (1.1), (1.3) respectively.

$f_{w}(x)=\frac{x^{c} f(x)}{E\left[x^{c}\right]}$

$f_{w}(x)=\frac{\theta^{c+1} x^{c}\left((\alpha+\theta)\left(2 \alpha+x^{2} \theta^{2}\right)+2 \theta(\theta-1)(1+\alpha x)\right) e^{-\theta x}}{c !((\theta+\alpha)(2 \alpha+(c+1)(c+2))+2(\theta-1)(\theta+\alpha(c+1)))} \quad x>0, \theta>0, \alpha \geq 0, c \geq 0$

Proof of (2.2) being a probability density function is given below

$$
\begin{aligned}
& \text { PROOF: } \int_{0}^{\infty} f_{w}(x)=\int_{0}^{\infty} \frac{\theta^{c+1} x^{c}\left((\alpha+\theta)\left(2 \alpha+x^{2} \theta^{2}\right)+2 \theta(\theta-1)(1+\alpha x)\right) e^{-\theta x}}{c !(\theta+\alpha)(2 \alpha+(c+1)(c+2))+2(\theta-1)(\theta+\alpha(c+1)))} \\
& =\frac{\theta^{c+1}}{c !((\theta+\alpha)(2 \alpha+(c+1)(c+2))+2(\theta-1)(\theta+\alpha(c+1)))} \int_{0}^{\infty} x^{c}\left((\alpha+\theta)\left(2 \alpha+x^{2} \theta^{2}\right)+2 \theta(\theta-1)(1+\alpha x)\right) e^{-\theta x} \\
& =\frac{\theta^{c+1}}{c !((\theta+\alpha)(2 \alpha+(c+1)(c+2))+2(\theta-1)(\theta+\alpha(c+1)))} \times \frac{c !((\theta+\alpha)(2 \alpha+(c+1)(c+2))+2(\theta-1)(\theta+\alpha(c+1)))}{\theta^{c+1}} \\
& =1 \\
& \int_{0}^{\infty} f_{w}(x)=1
\end{aligned}
$$


So (2.2) is a probability density function

where $\int_{0}^{\infty} x^{\beta-1} e^{-\alpha x} d x=\frac{\Gamma \beta}{\alpha^{\beta}}$ is a gamma function

The plots of p.d.f and c.d.f for different values of parameters are given in graphs $1 \&$ 2 below. Graph 1 indicating that proposed model is positively skewed \& platykurtic as well as leptokurtic. Graph 2 Indicating curve starts with zero and ends at one and showing c.d.f is non-decreasing function.

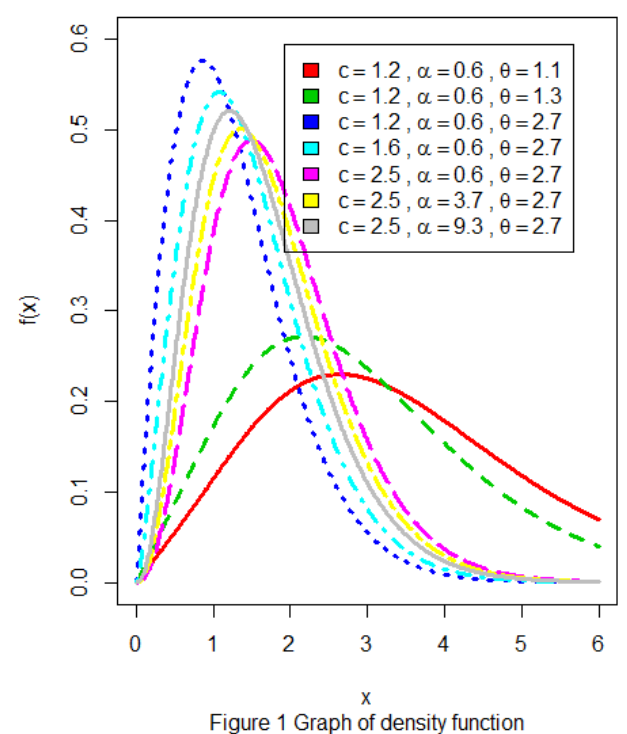

Figure 1 Graph of density function

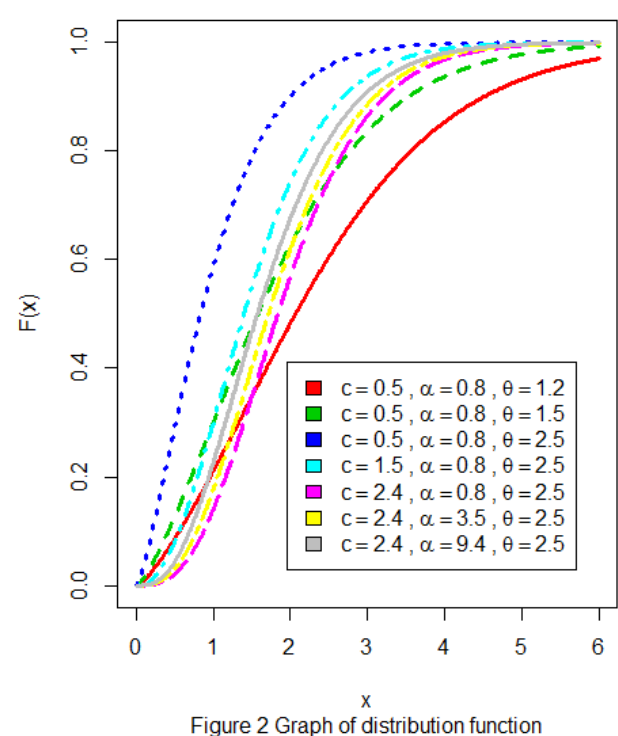

Figure 2 Graph of distribution function

The corresponding c.d.f of Weighted Lindley-Quasi Xgamma distribution is given in

(2.3) \& obtained as $F_{w}(x)=\int_{0}^{x} \frac{\theta^{c+1} x^{c}\left((\alpha+\theta)\left(2 \alpha+x^{2} \theta^{2}\right)+2 \theta(\theta-1)(1+\alpha x)\right) e^{-\theta x}}{c !(\theta+\alpha)(2 \alpha+(c+1)(c+2))+2(\theta-1)(\theta+\alpha(c+1)))} d x$

Put $\theta x=t$ in (2.3)

$\theta d x=d t$

as $x \rightarrow 0, t \rightarrow 0$ and $x \rightarrow x, t \rightarrow \theta x$ 
$F_{w}(x)=\left[\frac{(\alpha+\theta)(2 \alpha \gamma(c+1, \theta x)+\gamma(c+3, \theta x))+2(\theta-1)(\theta \gamma(c+1, \theta x)+\alpha \gamma(c+2, \theta x))}{c !((\theta+\alpha)(2 \alpha+(c+1)(c+2))+2(\theta-1)(\theta+\alpha(c+1)))}\right]$

where $\gamma(s, x)=\int_{0}^{x} t^{s-1} e^{-t} d t$ is a lower incomplete gamma function

\section{NEED OF PROPOSED MODEL AND MOTIVATION FOR DEVELOPING PROPOSED MODEL}

There are many situations in real life where models with less parameters don't perform better. Here in this paper we added an extra parameter known as weight parameter to two parameter Lindley-Quasi Xgamma distribution to obtain a generalized model which will find greater applicability in dealing with complex data. It can be observed from Table 1 that proposed model can be used for over dispersed as well as under dispersed data. Addition of weight parameter also brings the flexibility in terms of moments as can be seen from graphs of probability density function and hazard rate. Complex data applicability and increased flexibility were two important points which motivated us to work on this model.

\section{RELIABILITY MEASURES}

This division of paper presents survival function, hazard rate, reverse hazard rate of the proposed Weighted Lindley-Quasi Xgamma distribution for random variable $X$, where $X$ represents the lifetime of a system.

\subsection{Reliability Function $\mathrm{R}(\mathrm{x})$}

The reliability function or survival function $R(x)$ gives the numerical value of odds of surviving of a system beyond a specified time $(t)$.

Mathematically

$R_{w}(x)=P(X>t)=1-F_{w}(x)$

The reliability function or the survival function of Weighted Lindley-Quasi Xgamma distribution is obtained as:

$R_{w}(x)=1-\left[\frac{(\alpha+\theta)(2 \alpha \gamma(c+1, \theta x)+\gamma(c+3, \theta x))+2(\theta-1)(\theta \gamma(c+1, \theta x)+\alpha \gamma(c+2, \theta x))}{c !((\theta+\alpha)(2 \alpha+(c+1)(c+2))+2(\theta-1)(\theta+\alpha(c+1)))}\right]$ 


\subsection{Hazard Rate}

The hazard rate which is defined as chance that a system which is surviving up to time " $t$ " will fail in the small time interval after " $t$ " is obtained for WLQXD as:

$$
\begin{aligned}
& H . R=h_{w}(x)=\frac{f_{w}(x)}{R_{w}(x)} \\
& =\left(\frac{\theta^{c+1} x^{c}\left((\alpha+\theta)\left(2 \alpha+x^{2} \theta^{2}\right)+2 \theta(\theta-1)(1+\alpha x)\right) e^{-\theta x}}{\left(\begin{array}{l}
c !((\theta+\alpha)(2 \alpha+(c+1)(c+2))+2(\theta-1)(\theta+\alpha(c+1)))- \\
(\alpha+\theta)(2 \alpha \gamma(c+1, \theta x)+\gamma(c+3, \theta x))+2(\theta-1)(\theta \gamma(c+1, \theta x)+\alpha \gamma(c+2, \theta x))
\end{array}\right)}\right)
\end{aligned}
$$

The graphs of reliability function and hazard rate of WLQXD for different values of parameter are given below

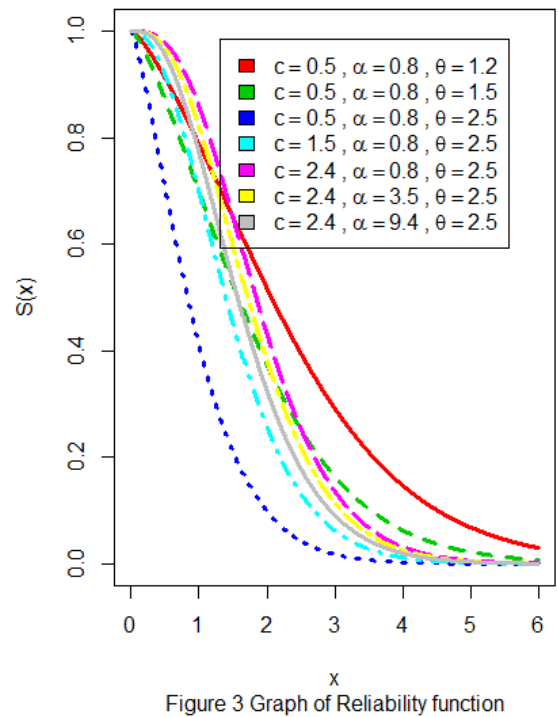

Figure 3 Graph of Reliability function

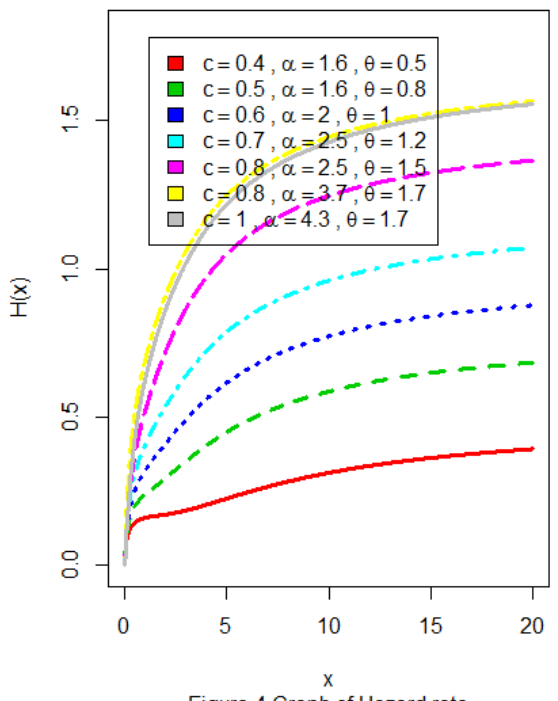

Figure 4 Graph of Hazard rate

From the above graph of hazard rate it is revealed that our proposed model possesses non-decreasing hazard rate and it can be also seen that hazard rate becomes constant as value of $x$ increases. There are many situations in real life where hazard rate is nondecreasing, like lifetime of human beings, animals etc. 


\subsection{Reverse Hazard Rate}

The reverse hazard rate of the Weighted Lindley-Quasi Xgamma distribution is given as:

$$
\begin{aligned}
& \text { R.H.R }=h_{r_{w}}(x)=\frac{f_{w}(x)}{F_{w}(x)} \\
& =\frac{\theta^{c+1} x^{c}\left((\alpha+\theta)\left(2 \alpha+x^{2} \theta^{2}\right)+2 \theta(\theta-1)(1+\alpha x)\right) e^{-\theta x}}{(\alpha+\theta)(2 \alpha \gamma(c+1, \theta x)+\gamma(c+3, \theta x))+2(\theta-1)(\theta \gamma(c+1, \theta x)+\alpha \gamma(c+2, \theta x))}
\end{aligned}
$$

\section{STATISTICAL PROPERTIES}

Moments, characteristic function, mean deviation, harmonic mean, coefficient of variation characterize probability models. Here we have obtained these statistical properties for proposed Weighted Lindley-Quasi Xgamma distribution.

\subsection{Moments and Related Measures}

Assuming $X$ to be a random variable having Weighted Lindley-Quasi Xgamma distribution with parameters $\theta, c$ and $\alpha$.Then the $r^{\text {th }}$ moment about origin for a given probability distribution is given by

$$
\begin{aligned}
& \mu_{r}{ }^{\prime}=E\left(X^{r}\right)=\int_{0}^{\infty} x^{r} f_{w}(x) d x \quad r=1,2,3 \ldots \\
& =\int_{0}^{\infty} x^{r} \frac{\theta^{c+1} x^{c}\left((\alpha+\theta)\left(2 \alpha+x^{2} \theta^{2}\right)+2 \theta(\theta-1)(1+\alpha x)\right) e^{-\theta x}}{c !((\theta+\alpha)(2 \alpha+(c+1)(c+2))+2(\theta-1)(\theta+\alpha(c+1)))} d x \\
& \mu_{r}^{\prime}=\left[\frac{(r+c) !((\alpha+\theta)(2 \alpha+(r+c+1)(r+c+2))+2(\theta-1)(\theta+\alpha(r+c+1)))}{\theta^{r}(c !((\theta+\alpha)(2 \alpha+(c+1)(c+2))+2(\theta-1)(\theta+\alpha(c+1))))}\right]
\end{aligned}
$$

Put $r=1$ in equation (5.1.1) we get

$$
\mu_{1}^{\prime}=\left[\frac{(c+1)((\alpha+\theta)(2 \alpha+(c+2)(c+3))+2(\theta-1)(\theta+\alpha(c+2)))}{\theta(((\theta+\alpha)(2 \alpha+(c+1)(c+2))+2(\theta-1)(\theta+\alpha(c+1))))}\right]
$$

Which is mean of the WLQXD. 
Put $r=2$ in equation (5.1.1) we get

$$
\mu_{2}^{\prime}=\left[\frac{(c+1)(c+2)((\alpha+\theta)(2 \alpha+(c+4)(c+3))+2(\theta-1)(\theta+\alpha(c+3)))}{\theta^{2}(((\theta+\alpha)(2 \alpha+(c+1)(c+2))+2(\theta-1)(\theta+\alpha(c+1))))}\right]
$$

The variance $\mu_{2}$ of WLQXD is

$$
\mu_{2}=\left(\begin{array}{l}
{\left[\frac{(c+1)(c+2)((\alpha+\theta)(2 \alpha+(c+4)(c+3))+2(\theta-1)(\theta+\alpha(c+3)))}{\theta^{2}(((\theta+\alpha)(2 \alpha+(c+1)(c+2))+2(\theta-1)(\theta+\alpha(c+1))))}\right]-} \\
{\left[\frac{(c+1)((\alpha+\theta)(2 \alpha+(c+2)(c+3))+2(\theta-1)(\theta+\alpha(c+2)))}{\theta(((\theta+\alpha)(2 \alpha+(c+1)(c+2))+2(\theta-1)(\theta+\alpha(c+1))))}\right]^{2}}
\end{array}\right)
$$

The coefficient of variation (C.V) of WLQXD is

$$
\begin{aligned}
& C . V=\frac{\left(\mu_{2}\right)^{\frac{1}{2}}}{\mu_{1}{ }^{\prime}} \\
& =\left(\begin{array}{l}
{\left[\frac{(c+1)(c+2)((\alpha+\theta)(2 \alpha+(c+4)(c+3))+2(\theta-1)(\theta+\alpha(c+3)))}{\theta^{2}(((\theta+\alpha)(2 \alpha+(c+1)(c+2))+2(\theta-1)(\theta+\alpha(c+1))))}\right]-} \\
{\left[\frac{(c+1)((\alpha+\theta)(2 \alpha+(c+2)(c+3))+2(\theta-1)(\theta+\alpha(c+2)))}{\theta(((\theta+\alpha)(2 \alpha+(c+1)(c+2))+2(\theta-1)(\theta+\alpha(c+1)))}\right]^{2}}
\end{array}\right)^{\frac{1}{2}} \\
& {\left[\frac{(c+1)((\alpha+\theta)(2 \alpha+(c+2)(c+3))+2(\theta-1)(\theta+\alpha(c+2)))}{\theta(((\theta+\alpha)(2 \alpha+(c+1)(c+2))+2(\theta-1)(\theta+\alpha(c+1))))}\right]}
\end{aligned}
$$

The index of dispersion $(\gamma)$ of WLQXD is

$$
\begin{aligned}
& \gamma=\frac{\left(\mu_{2}\right)}{\mu_{1}{ }^{\prime}} \\
& =\frac{\left(\begin{array}{l}
{\left[\frac{(c+1)(c+2)((\alpha+\theta)(2 \alpha+(c+4)(c+3))+2(\theta-1)(\theta+\alpha(c+3)))}{\theta^{2}(((\theta+\alpha)(2 \alpha+(c+1)(c+2))+2(\theta-1)(\theta+\alpha(c+1))))}\right]-} \\
{\left[\frac{(c+1)((\alpha+\theta)(2 \alpha+(c+2)(c+3))+2(\theta-1)(\theta+\alpha(c+2)))}{\theta(((\theta+\alpha)(2 \alpha+(c+1)(c+2))+2(\theta-1)(\theta+\alpha(c+1)))}\right]^{2}}
\end{array}\right)}{\left[\frac{(c+1)((\alpha+\theta)(2 \alpha+(c+2)(c+3))+2(\theta-1)(\theta+\alpha(c+2)))}{\theta(((\theta+\alpha)(2 \alpha+(c+1)(c+2))+2(\theta-1)(\theta+\alpha(c+1))))}\right]}
\end{aligned}
$$


Table 1: Description of WLQXD for different parameter values.

\begin{tabular}{|l|l|l|l|l|l|l|}
\hline$\alpha$ & $\theta$ & $c$ & Mean & Variance & $\begin{array}{l}\text { Index of } \\
\text { dispersion }\end{array}$ & $\begin{array}{l}\text { Coefficient } \\
\text { of variation }\end{array}$ \\
\hline 0.5 & 0.5 & 0.3 & 6.353521 & 14.5477 & 2.289707 & 0.6003198 \\
\hline 2.5 & 0.5 & 0.5 & 4.704545 & 14.00362 & 2.976614 & 0.7954308 \\
\hline 2.5 & 5.5 & 1.5 & 0.6199305 & 0.1368836 & 0.2208048 & 0.5968055 \\
\hline 0.5 & 5.5 & 2.0 & 0.7485493 & 0.1655399 & 0.2211476 & 0.5435393 \\
\hline
\end{tabular}

It can be observed from Table 1 that WLQXD is over dispersed as well as under dispersed for different parameter values.

\subsection{Harmonic Mean of Weighted Lindley-Quasi Xgamma Distribution}

The harmonic mean $H$ of the WLQXD is determined as

$$
\begin{aligned}
& \frac{1}{H}=E\left[\frac{1}{X}\right]=\int_{0}^{\infty} \frac{1}{x} f_{w}(x) d x \\
& =\int_{0}^{\infty} \frac{1}{x} \frac{\theta^{c+1} x^{c}\left((\alpha+\theta)\left(2 \alpha+x^{2} \theta^{2}\right)+2 \theta(\theta-1)(1+\alpha x)\right) e^{-\theta x}}{c !((\theta+\alpha)(2 \alpha+(c+1)(c+2))+2(\theta-1)(\theta+\alpha(c+1)))} d x \\
& H=\frac{c((\theta+\alpha)(2 \alpha+(c+1)(c+2))+2(\theta-1)(\theta+\alpha(c+1)))}{\theta((\alpha+\theta)(2 \alpha+c(c+1))+2(\theta-1)(\theta+\alpha c))}
\end{aligned}
$$

\subsection{Mean Deviation about Mean and Median of WLQXD}

We have derived the expressions for mean deviation about mean and median of WLQXD in this section.

THEOREM 1. If $X$ has the $\operatorname{WLQXD}(\theta, \alpha, c)$, then the mean deviation about mean $\left(\delta_{1}(X)\right)$ and mean deviation about median $\left(\delta_{2}(X)\right)$ are given as:

$$
\delta_{1}(X)=\left[\begin{array}{l}
2 \mu\left\{\frac{(\alpha+\theta)(2 \alpha \gamma(c+1, \theta \mu)+\gamma(c+3, \theta \mu))+2(\theta-1)(\theta \gamma(c+1, \theta \mu)+\alpha \gamma(c+2, \theta \mu))}{c !((\theta+\alpha)(2 \alpha+(c+1)(c+2))+2(\theta-1)(\theta+\alpha(c+1)))}\right\} \\
-2\left\{\frac{(\alpha+\theta)(2 \alpha \gamma(c+2, \theta \mu)+\gamma(c+4, \theta \mu))+2(\theta-1)(\theta \gamma(c+2, \theta \mu)+\alpha \gamma(c+3, \theta \mu))}{\theta c !((\theta+\alpha)(2 \alpha+(c+1)(c+2))+2(\theta-1)(\theta+\alpha(c+1)))}\right\}
\end{array}\right]
$$


And

$\delta_{2}(X)=\left[\mu-2\left\{\frac{(\alpha+\theta)(2 \alpha \gamma(c+2, \theta M)+\gamma(c+4, \theta M))+2(\theta-1)(\theta \gamma(c+2, \theta M)+\alpha \gamma(c+3, \theta M))}{\theta c !((\theta+\alpha)(2 \alpha+(c+1)(c+2))+2(\theta-1)(\theta+\alpha(c+1)))}\right\}\right]$

respectively.

PROOF. Mean deviation about mean and mean deviation about median are defined as

$\delta_{1}(X)=\int_{0}^{\infty}|x-\mu| f_{w}(x) d x$

And $\delta_{2}(X)=\int_{0}^{\infty}|x-M| f_{w}(x) d x$

respectively.

Where $\mu$ and $M$ are mean and median respectively of random variable $X \sim W L Q X D$.

The measures $\delta_{1}(X)$ and $\delta_{2}(X)$ can be obtained by using the simplified relationships.

$$
\begin{aligned}
& \delta_{1}(X)=\int_{0}^{\mu}(\mu-x) f_{w}(x) d x+\int_{\mu}^{\infty}(x-\mu) f_{w}(x) d x \\
& \delta_{1}(X)=2 \mu F_{w}(\mu)-2 \int_{0}^{\mu} x f_{w}(x) d x
\end{aligned}
$$

And

$$
\begin{aligned}
& \delta_{2}(X)=\int_{0}^{M}(M-x) f_{w}(x) d x+\int_{M}^{\infty}(x-M) f_{w}(x) d x \\
& \delta_{2}(X)=\mu-2 \int_{0}^{M} x f_{w}(x) d x
\end{aligned}
$$

Where $f_{w}(x)=\frac{\theta^{c+1} x^{c}\left((\alpha+\theta)\left(2 \alpha+x^{2} \theta^{2}\right)+2 \theta(\theta-1)(1+\alpha x)\right) e^{-\theta x}}{c !((\theta+\alpha)(2 \alpha+(c+1)(c+2))+2(\theta-1)(\theta+\alpha(c+1)))}$ 
Now

$$
\int_{0}^{\mu} x f_{w}(x) d x=\left[\frac{(\alpha+\theta)(2 \alpha \gamma(c+2, \theta \mu)+\gamma(c+4, \theta \mu))+2(\theta-1)(\theta \gamma(c+2, \theta \mu)+\alpha \gamma(c+3, \theta \mu))}{\theta c !((\theta+\alpha)(2 \alpha+(c+1)(c+2))+2(\theta-1)(\theta+\alpha(c+1)))}\right]
$$

And

$$
\int_{0}^{M} x f_{w}(x) d x=\left[\frac{(\alpha+\theta)(2 \alpha \gamma(c+2, \theta M)+\gamma(c+4, \theta M))+2(\theta-1)(\theta \gamma(c+2, \theta M)+\alpha \gamma(c+3, \theta M))}{\theta c !((\theta+\alpha)(2 \alpha+(c+1)(c+2))+2(\theta-1)(\theta+\alpha(c+1)))}\right]
$$

Using expressions (5.3.1), (5.3.2), (5.3.3) and (5.3.4) and expression for c.d.f (2.4) we obtain mean deviation about mean $\left(\delta_{1}(X)\right)$ and mean deviation about median

$$
\left(\delta_{2}(X)\right) \delta_{1}(X)=\left[\begin{array}{l}
2 \mu\left\{\frac{(\alpha+\theta)(2 \alpha \gamma(c+1, \theta \mu)+\gamma(c+3, \theta \mu))+2(\theta-1)(\theta \gamma(c+1, \theta \mu)+\alpha \gamma(c+2, \theta \mu))}{c !((\theta+\alpha)(2 \alpha+(c+1)(c+2))+2(\theta-1)(\theta+\alpha(c+1)))}\right\} \\
-2\left\{\frac{(\alpha+\theta)(2 \alpha \gamma(c+2, \theta \mu)+\gamma(c+4, \theta \mu))+2(\theta-1)(\theta \gamma(c+2, \theta \mu)+\alpha \gamma(c+3, \theta \mu))}{\theta c !((\theta+\alpha)(2 \alpha+(c+1)(c+2))+2(\theta-1)(\theta+\alpha(c+1)))}\right\}
\end{array}\right]
$$

$\&$

$$
\delta_{2}(X)=\left[\mu-2\left\{\frac{(\alpha+\theta)(2 \alpha \gamma(c+2, \theta M)+\gamma(c+4, \theta M))+2(\theta-1)(\theta \gamma(c+2, \theta M)+\alpha \gamma(c+3, \theta M))}{\theta c !((\theta+\alpha)(2 \alpha+(c+1)(c+2))+2(\theta-1)(\theta+\alpha(c+1)))}\right\}\right]
$$

\subsection{Generating functions of Weighted Lindley-Quasi Xgamma Distribution}

We will derive moment generating function and characteristic function of WLQXD in this segment of paper.

THEOREM 2. If $X \sim \operatorname{WLQXD}(\theta, \alpha, c)$ then the moment generating function $M_{X}(t)$ and characteristic generating function $\phi_{X}(t)$ are

$$
M_{X}(t)=\left(\begin{array}{l}
\frac{\theta^{c+1}}{((\theta+\alpha)(2 \alpha+(c+1)(c+2))+2(\theta-1)(\theta+\alpha(c+1)))} \\
\left((\alpha+\theta)\left(\frac{2 \alpha}{(\theta-t)^{c+1}}+\frac{\theta^{2}(c+1)(c+2)}{(\theta-t)^{c+3}}\right)+2 \theta(\theta-1)\left(\frac{1}{(\theta-t)^{c+1}}+\frac{(c+1) \alpha}{(\theta-t)^{c+2}}\right)\right)
\end{array}\right)
$$


And

$$
\phi_{X}(t)=\left(\begin{array}{l}
\frac{\theta^{\mathrm{c}+1}}{((\theta+\alpha)(2 \alpha+(c+1)(c+2))+2(\theta-1)(\theta+\alpha(c+1)))} \\
\left((\alpha+\theta)\left(\frac{2 \alpha}{(\theta-i t)^{c+1}}+\frac{\theta^{2}(c+1)(c+2)}{(\theta-i t)^{c+3}}\right)+2 \theta(\theta-1)\left(\frac{1}{(\theta-i t)^{c+1}}+\frac{(c+1) \alpha}{(\theta-i t)^{c+2}}\right)\right)
\end{array}\right)
$$

respectively.

PROOF. We begin with the well-known definition of the moment generating function given by

$$
\begin{aligned}
& M_{X}(t)=E\left(e^{t X}\right)=\int_{0}^{\infty} e^{t x} f_{w}(x) d x \\
& =\int_{0}^{\infty} e^{t x} \frac{\theta^{c+1} x^{c}\left((\alpha+\theta)\left(2 \alpha+x^{2} \theta^{2}\right)+2 \theta(\theta-1)(1+\alpha x)\right) e^{-\theta x}}{c !((\theta+\alpha)(2 \alpha+(c+1)(c+2))+2(\theta-1)(\theta+\alpha(c+1)))} d x \\
& M_{X}(t)=\left(\frac{\theta^{c+1}}{((\theta+\alpha)(2 \alpha+(c+1)(c+2))+2(\theta-1)(\theta+\alpha(c+1)))}\right. \\
& \left.\left((\alpha+\theta)\left(\frac{2 \alpha}{(\theta-t)^{c+1}}+\frac{\theta^{2}(c+1)(c+2)}{(\theta-t)^{c+3}}\right)+2 \theta(\theta-1)\left(\frac{1}{(\theta-t)^{c+1}}+\frac{(c+1) \alpha}{(\theta-t)^{c+2}}\right)\right)\right)
\end{aligned}
$$

Which is the m.g.f of Weighted Lindley-Quasi Xgamma distribution.

Also we know that $\phi_{X}(t)=M_{X}(i t)$

Therefore,

$$
\phi_{X}(t)=\left(\begin{array}{l}
\frac{\theta^{c+1}}{((\theta+\alpha)(2 \alpha+(c+1)(c+2))+2(\theta-1)(\theta+\alpha(c+1)))} \\
\left((\alpha+\theta)\left(\frac{2 \alpha}{(\theta-i t)^{c+1}}+\frac{\theta^{2}(c+1)(c+2)}{(\theta-i t)^{c+3}}\right)+2 \theta(\theta-1)\left(\frac{1}{(\theta-i t)^{c+1}}+\frac{(c+1) \alpha}{(\theta-i t)^{c+2}}\right)\right)
\end{array}\right)
$$

Which is the characteristic function of WLQXD distribution. 


\section{ORDER STATISTICS OF WEIGHTED LINDLEY-QUASI XGAMMA DISTRIBUTION}

Consider $X_{(1)}, X_{(2)}, X_{(3)} \ldots, X_{(n)}$ to be the ordered statistics of the random sample $x_{1}, x_{2}, x_{3}, \ldots x_{n}$ obtained from the Weighted Lindley-Quasi Xgamma distribution with cumulative distribution function $F_{w}(x)$ and probability density function $f_{w}(x)$, then the probability density function of $v^{\text {th }}$ order statistics $X_{(v)}$ is given by:

$$
f_{v w}(x)=\frac{n !}{(v-1) !(n-v) !} f_{w}(x)\left[F_{w}(x)\right]^{p-1}\left[1-F_{w}(x)\right]^{n-v} \quad v=1,2,3 \ldots n
$$

Using the equations (2.2) and (2.3), the probability density function of $v^{\text {th }}$ order statistics of Weighted Lindley-Quasi Xgamma distribution is given by:

$$
f_{(v) w}(x)=\left[\begin{array}{l}
\frac{n !}{(v-1) !(n-v) !}\left\{\frac{\theta^{c+1} x^{c}\left((\alpha+\theta)\left(2 \alpha+x^{2} \theta^{2}\right)+2 \theta(\theta-1)(1+\alpha x)\right) e^{-\theta x}}{c !((\theta+\alpha)(2 \alpha+(c+1)(c+2))+2(\theta-1)(\theta+\alpha(c+1)))}\right\} \\
{\left[\frac{(\alpha+\theta)(2 \alpha \gamma(c+1, \theta x)+\gamma(c+3, \theta x))+2(\theta-1)(\theta \gamma(c+1, \theta x)+\alpha \gamma(c+2, \theta x))}{c !((\theta+\alpha)(2 \alpha+(c+1)(c+2))+2(\theta-1)(\theta+\alpha(c+1)))}\right]^{v-1}} \\
{\left[1-\left(\frac{(\alpha+\theta)(2 \alpha \gamma(c+1, \theta x)+\gamma(c+3, \theta x))+2(\theta-1)(\theta \gamma(c+1, \theta x)+\alpha \gamma(c+2, \theta x))}{c !((\theta+\alpha)(2 \alpha+(c+1)(c+2))+2(\theta-1)(\theta+\alpha(c+1)))}\right)\right]^{n-v}}
\end{array}\right]
$$

Then, the p.d.f of first order statistic $X_{(1)}$ of Weighted Lindley-Quasi Xgamma distribution is given by:

$$
f_{(1) w}(x)=\left[\begin{array}{l}
n\left\{\frac{\theta^{c+1} x^{c}\left((\alpha+\theta)\left(2 \alpha+x^{2} \theta^{2}\right)+2 \theta(\theta-1)(1+\alpha x)\right) e^{-\theta x}}{c !((\theta+\alpha)(2 \alpha+(c+1)(c+2))+2(\theta-1)(\theta+\alpha(c+1)))}\right\} \\
\left.\left[1-\left(\frac{(\alpha+\theta)(2 \alpha \gamma(c+1, \theta x)+\gamma(c+3, \theta x))+2(\theta-1)(\theta \gamma(c+1, \theta x)+\alpha \gamma(c+2, \theta x))}{c !((\theta+\alpha)(2 \alpha+(c+1)(c+2))+2(\theta-1)(\theta+\alpha(c+1)))}\right)\right]^{n-1} \cdot\right]
\end{array}\right]
$$

and the pdf of $n^{\text {th }}$ order statistic $X_{(n)}$ of Weighted Lindley-Quasi Xgamma distribution is given as: 


$$
f_{(n) w}(x)=\left[\begin{array}{l}
n\left\{\frac{\theta^{c+1} x^{c}\left((\alpha+\theta)\left(2 \alpha+x^{2} \theta^{2}\right)+2 \theta(\theta-1)(1+\alpha x)\right) e^{-\theta x}}{c !((\theta+\alpha)(2 \alpha+(c+1)(c+2))+2(\theta-1)(\theta+\alpha(c+1)))}\right\} \\
\left.\left[\frac{(\alpha+\theta)(2 \alpha \gamma(c+1, \theta x)+\gamma(c+3, \theta x))+2(\theta-1)(\theta \gamma(c+1, \theta x)+\alpha \gamma(c+2, \theta x))}{c !((\theta+\alpha)(2 \alpha+(c+1)(c+2))+2(\theta-1)(\theta+\alpha(c+1)))}\right]^{n-1} \cdot\right]
\end{array}\right]
$$

\section{BONFERRONI AND LORENZ CURVES AND INDICES OF WLQXD}

The Bonferroni curve $(B(p))$, Lorenz curve $(L(p))$, Bonferroni index $(B)$ and Gini index $(G)$ have find applicability in fields of economics, demography, reliability, life testing and medical sciences. The Bonferroni and Lorenz curves are defined as

$$
\begin{aligned}
& B(p)=\frac{1}{p \mu} \int_{0}^{q} x f_{w}(x) d x \\
& L(p)=\frac{1}{\mu} \int_{0}^{q} x f_{w}(x) d x
\end{aligned}
$$

Where $\mu=E(x)$ is the mean of WLQXD and $q=F^{-1}(p)$.

The Bonferroni and Gini indices are defined as

$$
\begin{aligned}
& B=1-\int_{0}^{1} B(p) d p \\
& G=1-2 \int_{0}^{1} L(p) d p
\end{aligned}
$$

Using the p.d.f (2.2) of WLQXD we get

$$
\int_{0}^{q} x f_{w}(x) d x=\left[\frac{(\alpha+\theta)(2 \alpha \gamma(c+2, \theta q)+\gamma(c+4, \theta q))+2(\theta-1)(\theta \gamma(c+2, \theta q)+\alpha \gamma(c+3, \theta q))}{\theta c !((\theta+\alpha)(2 \alpha+(c+1)(c+2))+2(\theta-1)(\theta+\alpha(c+1)))}\right]
$$


Using equation (7.5) in (7.1) \& (7.2) we get

$$
B(p)=\frac{1}{p \mu}\left[\frac{(\alpha+\theta)(2 \alpha \gamma(c+2, \theta q)+\gamma(c+4, \theta q))+2(\theta-1)(\theta \gamma(c+2, \theta q)+\alpha \gamma(c+3, \theta q))}{\theta c !((\theta+\alpha)(2 \alpha+(c+1)(c+2))+2(\theta-1)(\theta+\alpha(c+1)))}\right]
$$

And

$$
L(p)=\frac{1}{\mu}\left[\frac{(\alpha+\theta)(2 \alpha \gamma(c+2, \theta q)+\gamma(c+4, \theta q))+2(\theta-1)(\theta \gamma(c+2, \theta q)+\alpha \gamma(c+3, \theta q))}{\theta c !((\theta+\alpha)(2 \alpha+(c+1)(c+2))+2(\theta-1)(\theta+\alpha(c+1)))}\right]
$$

Using (7.6) \& (7.7) in (7.3) \& (7.4) we get

$$
\begin{aligned}
& B=1-\frac{1}{\mu}\left[\frac{(\alpha+\theta)(2 \alpha \gamma(c+2, \theta q)+\gamma(c+4, \theta q))+2(\theta-1)(\theta \gamma(c+2, \theta q)+\alpha \gamma(c+3, \theta q))}{\theta c !((\theta+\alpha)(2 \alpha+(c+1)(c+2))+2(\theta-1)(\theta+\alpha(c+1)))}\right] \\
& L=1-\frac{2}{\mu}\left[\frac{(\alpha+\theta)(2 \alpha \gamma(c+2, \theta q)+\gamma(c+4, \theta q))+2(\theta-1)(\theta \gamma(c+2, \theta q)+\alpha \gamma(c+3, \theta q))}{\theta c !((\theta+\alpha)(2 \alpha+(c+1)(c+2))+2(\theta-1)(\theta+\alpha(c+1)))}\right]
\end{aligned}
$$

\section{RENYI ENTROPY}

The information associated with various values of random variable following a particular distribution is called entropy. Renyi entropy $T_{R}(\delta)$ of random variable X following Weighted Lindley-Quasi Xgamma distribution is obtained as

$$
T_{R}(\gamma)=\frac{1}{1-\delta} \log \left(\int_{0}^{\infty} f_{w} \delta(x) d x\right)
$$

Where $\delta>0$ and $\delta \neq 1$

$$
\begin{aligned}
& T_{R}(\delta)=\frac{1}{1-\delta} \log \left(\int_{0}^{\infty}\left(\frac{\theta^{c+1} x^{c}\left((\alpha+\theta)\left(2 \alpha+x^{2} \theta^{2}\right)+2 \theta(\theta-1)(1+\alpha x)\right) e^{-\theta x}}{c !((\theta+\alpha)(2 \alpha+(c+1)(c+2))+2(\theta-1)(\theta+\alpha(c+1)))}\right)^{\delta} d x\right) \\
& T_{R}(\delta)=\frac{1}{1-\delta} \log \left(\int_{0}^{\infty} \frac{\theta^{\delta(c+1)} x^{\delta c}\left((\alpha+\theta)\left(2 \alpha+x^{2} \theta^{2}\right)+2 \theta(\theta-1)(1+\alpha x)\right)^{\delta} e^{-\delta \theta x}}{(c !((\theta+\alpha)(2 \alpha+(c+1)(c+2))+2(\theta-1)(\theta+\alpha(c+1))))^{\delta}} d x\right)
\end{aligned}
$$


Using the binomial expansion

$$
\begin{aligned}
& \left((\alpha+\theta)\left(2 \alpha+x^{2} \theta^{2}\right)+2 \theta(\theta-1)(1+\alpha x)\right)^{\delta} \\
& =\sum_{k=0}^{\delta}\left(\begin{array}{l}
\delta \\
k
\end{array}\right) 2^{k} \theta^{k}(\theta-1)^{k}(1+\alpha x)^{k}(\alpha+\theta)^{\delta-k}\left(2 \alpha+x^{2} \theta^{2}\right)^{\delta-k} \\
& =\sum_{k=0}^{\delta}\left(\begin{array}{l}
\delta \\
k
\end{array}\right) 2^{k} \theta^{k}(\theta-1)^{k}(\alpha+\theta)^{\delta-k} \sum_{p=0}^{k}\left(\begin{array}{l}
k \\
p
\end{array}\right) \alpha^{p} x^{p} \sum_{l=0}^{\delta-k}\left(\begin{array}{l}
\delta-k \\
l
\end{array}\right) x^{2 l} \theta^{2 l} 2^{\delta-k-l} \alpha^{\delta-k-l}
\end{aligned}
$$

Using (8.2) in (8.1) we get

$$
\begin{aligned}
& T_{R}(\delta)=\frac{1}{1-\delta} \log \left\{\frac{\left(\begin{array}{l}
\delta \\
k=0
\end{array}\left(\begin{array}{l}
\delta \\
k
\end{array}\right)(\theta-1)^{k}(\alpha+\theta)^{\delta-k} \sum_{p=0}^{k}\left(\begin{array}{l}
k \\
p
\end{array}\right) \sum_{l=0}^{\delta-k}\left(\begin{array}{l}
\delta-k \\
l
\end{array}\right) \theta^{c \delta+\delta+k+2 l} 2^{\delta-l} \alpha^{p+\delta-k-l} \int_{0}^{\infty} x^{c \delta+p+2 l} e^{-\delta \theta x} d x\right)}{(c !((\theta+\alpha)(2 \alpha+(c+1)(c+2))+2(\theta-1)(\theta+\alpha(c+1))))^{\delta}}\right\}
\end{aligned}
$$

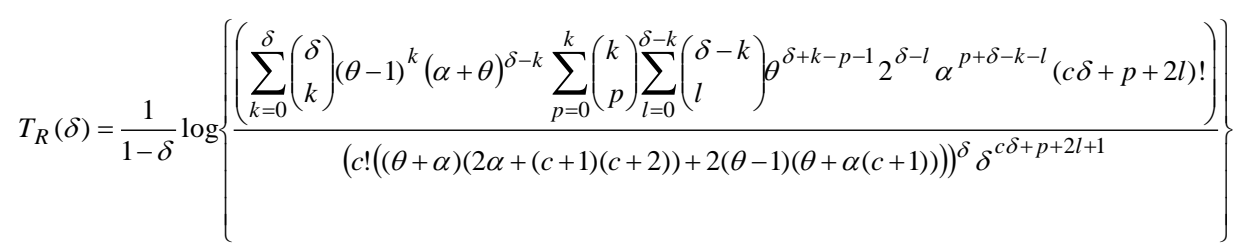

\section{ESTIMATION OF PARAMETERS OF WEIGHTED LINDLEY-QUASI- XGAMMA DISTRIBUTION}

Parameters are estimated by using method of maximum likelihood estimation. Considering $x_{1}, x_{2}, \ldots x_{n}$ to be the random sample of size $\mathrm{n}$ drawn from Weighted Lindley-Quasi Xgamma distribution having density function given by (2.2), then the likelihood function of WLQXD is given as:

$$
L(x \mid \theta, \alpha, c)=\prod_{i=1}^{n}\left(\frac{\theta^{c+1} x_{i}{ }^{c}\left((\alpha+\theta)\left(2 \alpha+x_{i}^{2} \theta^{2}\right)+2 \theta(\theta-1)\left(1+\alpha x_{i}\right)\right) e^{-\theta x_{i}}}{c !((\theta+\alpha)(2 \alpha+(c+1)(c+2))+2(\theta-1)(\theta+\alpha(c+1)))}\right)
$$

Taking log on both sides of likelihood function we get log likelihood function as: 


$$
\log L=\left(\begin{array}{l}
n(c+1) \log \theta+c \sum_{i=1}^{n} \log x_{i}-\theta \sum_{i=1}^{n} x_{i}+\sum_{i=1}^{n} \log \left((\alpha+\theta)\left(2 \alpha+x_{i}^{2} \theta^{2}\right)+2 \theta(\theta-1)\left(1+\alpha x_{i}\right)\right) \\
n \log c !-n \log ((\theta+\alpha)(2 \alpha+(c+1)(c+2))+2(\theta-1)(\theta+\alpha(c+1)))
\end{array}\right)
$$

Now partially differentiate (9.1) with respect to $\theta, \alpha, c$ and equating the result to zero, we obtain the following normal equations,

$$
\begin{aligned}
& \frac{\partial \log L}{\partial \theta} \\
& =\left[\begin{array}{l}
\frac{(c+1) n}{\theta}-\frac{n(2 \alpha+(c+1)(c+2)+4 \theta+2 \alpha(c+1)-2}{((\theta+\alpha)(2 \alpha+(c+1)(c+2))+2(\theta-1)(\theta+\alpha(c+1)))}+ \\
\sum_{i=1}^{n}\left(\frac{2 \alpha \theta x_{i}{ }^{2}+2 \alpha+3 x_{i}{ }^{2} \theta^{2}+2\left(1+\alpha x_{i}\right)(2 \theta-1)}{\left((\alpha+\theta)\left(2 \alpha+x_{i}{ }^{2} \theta^{2}\right)+2 \theta(\theta-1)\left(1+\alpha x_{i}\right)\right)}\right)-\sum_{i=1}^{n} x_{i}
\end{array}\right]=0 \\
& =\left[\begin{array}{l}
-\frac{n(2 \theta+(c+1)(c+2)+4 \alpha+2 \theta(c+1)-2(c+1)}{((\theta+\alpha)(2 \alpha+(c+1)(c+2))+2(\theta-1)(\theta+\alpha(c+1)))}+ \\
\sum_{i=1}^{n}\left(\frac{4 \alpha+x_{i}^{2} \theta^{2}+2 \theta+2 \theta(\theta-1) x_{i}}{\left((\alpha+\theta)\left(2 \alpha+x_{i}{ }^{2} \theta^{2}\right)+2 \theta(\theta-1)\left(1+\alpha x_{i}\right)\right)}\right)
\end{array}\right]=0 \\
& =\left[n \log \theta-\frac{n((\theta+\alpha)(2 c+3)+(2 \alpha \theta-2 \alpha))}{((\theta+\alpha)(2 \alpha+(c+1)(c+2))+2(\theta-1)(\theta+\alpha(c+1)))}-n\left(\log (c+1)-\frac{1}{2(c+1)}\right)+\sum_{i=1}^{n} \log x_{i}\right]=0
\end{aligned}
$$

MLEs of $\theta, \alpha, c$ cannot be obtained by solving above complex equations as these equations are not in closed form. So we solve above equations by using iteration method through R software. 


\section{SIMULATION STUDY}

In this part of paper we have carried out the simulation study for checking the performance of maximum likelihood (ML) estimates by taking different sample sizes $(n=30,50,70,110)$. We have used the inverse CDF technique for data simulation for WLQXD using R software. The process was repeated 1000 times for calculation of bias, variance \& mean square error (MSE) as are given values in Table 2. For two parameter combinations of WLQXD, decreasing trend is being observed in average bias, variance and MSE as we increase the sample size. Hence, the performance of ML estimators is quite good and consistent in case of Weighted Lindley-Quasi Xgamma Distribution.

Table 2: Simulation Study of ML estimators for WLQXD.

\begin{tabular}{|c|c|c|c|c|c|c|c|}
\hline \multirow{2}{*}{ Parameter } & \multirow{2}{*}{$\begin{array}{l}\text { n } \\
\text { (sample } \\
\text { size) }\end{array}$} & \multicolumn{3}{|c|}{$\alpha=0.8, \theta=0.7, c=0.8$} & \multicolumn{3}{|c|}{$\alpha=0.5, \theta=1.7, c=0.6$} \\
\hline & & Bias & Variance & MSE & Bias & Variance & MSE \\
\hline$\alpha$ & \multirow{3}{*}{30} & 0.4862598 & 0.4406534 & 0.677102 & 0.40865 & 0.540489 & 0.7074845 \\
\hline$\theta$ & & 1.625651 & 2.056811 & 4.699553 & 0.6685304 & 1.925161 & 2.372094 \\
\hline$c$ & & 0.4383506 & 0.5523539 & 0.7445051 & 0.5508202 & 0.5480579 & 0.8514608 \\
\hline$\alpha$ & \multirow[b]{3}{*}{50} & 0.478908 & 0.438007 & 0.6673606 & 0.36540 & 0.454492 & 0.5880101 \\
\hline$\theta$ & & 1.434994 & 0.9519631 & 3.011171 & 0.4003302 & 1.001894 & 1.162159 \\
\hline$c$ & & 0.3290241 & 0.2367339 & 0.3449908 & 0.4239412 & 0.2483344 & 0.4280606 \\
\hline$\alpha$ & \multirow{3}{*}{70} & 0.467299 & 0.4364103 & 0.6547787 & 0.30907 & 0.447506 & 0.5430311 \\
\hline$\theta$ & & 1.261992 & 0.6351194 & 2.227743 & 0.338809 & 0.6839647 & 0.7987562 \\
\hline$c$ & & 0.2534664 & 0.1573207 & 0.2215659 & 0.3918621 & 0.1738424 & 0.3273983 \\
\hline$\alpha$ & \multirow{3}{*}{110} & 0.459871 & 0.4333168 & 0.6447982 & 0.26780 & 0.398899 & 0.4706166 \\
\hline$\theta$ & & 1.17978 & 0.3789148 & 1.770795 & 0.2331814 & 0.4179511 & 0.4723247 \\
\hline$c$ & & 0.2156018 & 0.09346848 & 0.1399526 & 0.3397029 & 0.1026403 & 0.2180383 \\
\hline
\end{tabular}




\section{TESTING SIGNIFICANCE OF WEIGHT PARAMETER ON BASIS OF SIMULATED DATA FROM WLQXD}

For comparing proposed model with base model and for testing the significance of weighted parameter we generated random samples of sizes $(50,100,200,500)$ from WLQXD using inverse CDF technique. It is evident from the Table 3 that weighted parameter plays a highly significant role for large samples. Even though in small samples, the AIC, AICC, BIC and Negative Log likelihood values are also minimum in case of Weighted model as compared to base model. LR statistic for testing $H_{0}$ versus $H_{1}$ is $\psi=2\left(L(\hat{\Theta})-L\left(\hat{\Theta}_{0}\right)\right)$, where $\hat{\Theta}$ and $\hat{\Theta}_{0}$ are the MLEs under $H_{1}$ and $H_{0}$. The statistic $\psi$ is asymptotically (as $n \rightarrow \infty$ ) distributed as $\chi_{k}^{2}$, with $\mathrm{k}$ degrees of freedom which is equal to the difference in dimensionality of $\hat{\Theta}$ and $\hat{\Theta}_{0} . H_{0}$ will be rejected if the LR-test p-value is $<0.01$ (or LR Statistic value $>3.841$ ) at $95 \%$ confidence level

Table 3: Model Comparison Based On Simulated Data from WLQXD.

\begin{tabular}{|c|c|c|c|c|c|c|}
\hline \multicolumn{4}{|c|}{$\hat{c}=0.6, \hat{\alpha}=0.9, \hat{\theta}=1.5$} & \multicolumn{2}{|c|}{ Parameter Estimates } & \multirow{2}{*}{$\begin{array}{l}\text { Likelihood } \\
\text { Ratio } \\
\text { Statistic }\end{array}$} \\
\hline Criterion & WLQXD & LQXD & $\begin{array}{l}\text { Sample } \\
\text { Size (n) }\end{array}$ & WLQXD & LQXD & \\
\hline$-\log L$ & 13.02580 & 17.33376 & \multirow{4}{*}{50} & \multirow{4}{*}{$\begin{array}{l}\hat{c}=0.7756921 \\
(0.3682644) \\
\hat{\alpha}=0.7541315 \\
(3.0450987) \\
\hat{\theta}=4.8614277 \\
(0.9328238)\end{array}$} & \multirow{4}{*}{$\begin{array}{l}\hat{\alpha}=1.317147 \\
(1.466524) \\
\hat{\theta}=3.107664 \\
(0.352400)\end{array}$} & \multirow{4}{*}{8.615} \\
\hline AIC & 32.05161 & 38.66752 & & & & \\
\hline AICC & 24.05161 & 32.66752 & & & & \\
\hline BIC & 37.78767 & 42.49157 & & & & \\
\hline$-\log L$ & 19.48443 & 31.00223 & \multirow{4}{*}{100} & \multirow{4}{*}{$\begin{array}{l}\hat{c}=0.9303426 \\
(0.2523067) \\
\hat{\alpha}=1.9131216 \\
(3.0526314) \\
\hat{\theta}=5.3480262 \\
(0.6873024)\end{array}$} & \multirow{4}{*}{$\begin{array}{l}\hat{\alpha}=1.6114539 \\
(1.1262244) \\
\hat{\theta}=3.1965086 \\
(0.2635093)\end{array}$} & \multirow{4}{*}{23.035} \\
\hline AIC & 44.96885 & 66.00447 & & & & \\
\hline AICC & 36.96885 & 60.00447 & & & & \\
\hline BIC & 52.78436 & 71.21481 & & & & \\
\hline
\end{tabular}




\begin{tabular}{|c|c|c|c|c|c|c|}
\hline$-\log L$ & 33.61176 & 61.80406 & \multirow{4}{*}{200} & $\hat{c}=1.1748909$ & \multirow{4}{*}{$\begin{array}{l}\hat{\alpha}=1.5711807 \\
(0.7844924) \\
\hat{\theta}=3.2011676 \\
(0.1854941)\end{array}$} & \multirow{4}{*}{56.384} \\
\hline AIC & 73.22353 & 127.60812 & & (0.2734827). & & \\
\hline AICC & 65.22353 & 121.6081 & & (1.9152323) & & \\
\hline BIC & 83.11848 & 134.2048 & & $\begin{array}{l}\hat{\theta}=5.9318359 \\
(0.6560791)\end{array}$ & & \\
\hline$-\log L$ & 106.5939 & 134.1149 & \multirow{4}{*}{500} & $\hat{c}=0.54764349$ & \multirow{4}{*}{$\begin{array}{l}\hat{\alpha}=1.5740119 \\
(0.5568915) \\
\hat{\theta}=3.2970302 \\
(0.1221850)\end{array}$} & \multirow{4}{*}{55.042} \\
\hline AIC & 219.1878 & 272.2297 & & $(0.08917304)$ & & \\
\hline AICC & 211.1878 & 266.2297 & & $(1.05332253)$ & & \\
\hline BIC & 231.8316 & 280.6589 & & $\begin{array}{l}\hat{\theta}=4.64187400 \\
(0.26435388)\end{array}$ & & \\
\hline
\end{tabular}

\section{APPLICATIONS OF WEIGHTED LINDLEY-QUASI XGAMMA DISTRIBUTION}

Proposed model, its base model and some other lifetime models are fitted to two real life data sets for testing how fine proposed model fits to real life data sets as compared to other mentioned models.

DATA SET 1. The data set given in Table 4 represents the tensile strength measures in GPa of 69 carbon fibres tested under tension at gauge lengths of $20 \mathrm{~mm}$ and has been taken from Bader and Priest [10].

Table 4: Tensile strength measures in GPa of 69 carbon fibres.

\begin{tabular}{|l|l|l|l|l|l|l|l|}
\hline 1.312 & 1.314 & 1.479 & 1.552 & 1.700 & 1.803 & 1.861 & 1.865 \\
\hline 1.944 & 1.958 & 1.966 & 1.997 & 2.006 & 2.021 & 2.027 & 2.055 \\
\hline 2.063 & 2.098 & 2.140 & 2.179 & 2.224 & 2.240 & 2.253 & 2.270 \\
\hline 2.272 & 2.274 & 2.301 & 2.301 & 2.359 & 2.382 & 2.382 & 2.426 \\
\hline 2.434 & 2.435 & 2.478 & 2.490 & 2.511 & 2.514 & 2.535 & 2.554 \\
\hline 2.566 & 2.570 & 2.586 & 2.629 & 2.633 & 2.642 & 2.648 & 2.684 \\
\hline 2.697 & 2.726 & 2.770 & 2.773 & 2.800 & 2.809 & 2.818 & 2.821 \\
\hline 2.848 & 2.880 & 2.954 & 3.012 & 3.067 & 3.084 & 3.090 & 3.096 \\
\hline 3.128 & 3.233 & 3.433 & 3.585 & 3.585 & & & \\
\hline
\end{tabular}


DATA SET 2. This data set given in Table 5 is due to Smith and Naylor [11] consists of 63 observations of the strengths of $1.5 \mathrm{~cm}$ glass fibres, originally obtained by workers at the UK National Physical Laboratory.

Table 5: The strengths of $1.5 \mathrm{~cm}$ glass fibres of 63 observations.

\begin{tabular}{|l|l|l|l|l|l|l|l|}
\hline 0.55 & 0.74 & 0.77 & 0.81 & 0.84 & 0.93 & 1.04 & 1.11 \\
\hline 1.13 & 1.24 & 1.25 & 1.27 & 1.28 & 1.29 & 1.30 & 1.36 \\
\hline 1.39 & 1.42 & 1.48 & 1.48 & 1.49 & 1.49 & 1.50 & 1.50 \\
\hline 1.51 & 1.52 & 1.53 & 1.54 & 1.55 & 1.55 & 1.58 & 1.59 \\
\hline 1.60 & 1.61 & 1.61 & 1.61 & 1.61 & 1.62 & 1.62 & 1.63 \\
\hline 1.64 & 1.66 & 1.66 & 1.66 & 1.67 & 1.68 & 1.68 & 1.69 \\
\hline 1.70 & 1.70 & 1.73 & 1.76 & 1.76 & 1.77 & 1.78 & 1.81 \\
\hline 1.82 & 1.84 & 1.84 & 1.89 & 2.00 & 2.01 & 2.24 & \\
\hline
\end{tabular}

For the analysis of both the data sets we used R Software version 3.5.3.

Table 6: Summary Statistic of data set 1 and 2.

\begin{tabular}{|l|l|l|l|l|l|l|l|}
\hline $\begin{array}{l}\text { Data } \\
\text { set }\end{array}$ & $\begin{array}{l}\text { Number of } \\
\text { observations }\end{array}$ & Min. & Mean & $\begin{array}{l}\text { First } \\
\text { Quartile }\end{array}$ & Median & $\begin{array}{l}\text { Third } \\
\text { Quartile }\end{array}$ & Max. \\
\hline 1 & 69 & 1.312 & 2.451 & 2.098 & 2.478 & 2.773 & 3.585 \\
\hline 2 & 63 & 0.550 & 1.507 & 1.375 & 1.590 & 1.685 & 2.240 \\
\hline
\end{tabular}

Table 7: Model function of fitted models.

\begin{tabular}{|l|l|}
\hline Distribution & Model function \\
\hline $\begin{array}{l}\text { Weighted Lindley Quasi } \\
\text { Xamma Distribution }\end{array}$ & $\frac{\theta^{c+1} x^{c}\left((\alpha+\theta)\left(2 \alpha+x^{2} \theta^{2}\right)+2 \theta(\theta-1)(1+\alpha x)\right) e^{-\theta x}}{c !((\theta+\alpha)(2 \alpha+(c+1)(c+2))+2(\theta-1)(\theta+\alpha(c+1)))}$ \\
\hline $\begin{array}{l}\text { Lindley Quasi Xgamma } \\
\text { Distribution (LQXD) }\end{array}$ & $\frac{e^{-\theta x}}{(\alpha+\theta)^{2}}\left\{(\alpha+\theta)\left(\alpha+\frac{\mathrm{x}^{2} \theta^{2}}{2}\right)+\theta(\theta-1)(1+\alpha x)\right\}$ \\
\hline $\begin{array}{l}\text { Quasi Lindley Distribution } \\
\text { (QLD) }\end{array}$ & $\frac{\theta(\alpha+\theta x)}{\alpha+1} e^{-\theta x}$ \\
\hline $\begin{array}{l}\text { Exponential Distribution (ED) } \\
\text { Quasi Akash Distribution } \\
\text { (QAD) }\end{array}$ & $\frac{1}{\theta} e^{-\frac{x}{\theta}}$ \\
\hline
\end{tabular}


Table 8: ML estimates, -logL, AIC, AICC, BIC, HQIC, KS-distance, for fitted WLQXD and other mentioned models for data set 1.

\begin{tabular}{|l|l|l|l|l|l|}
\hline Distribution & WLQXD & LQXD & QLD & ED & QAD \\
\hline -logL & 49.9985 & 96.20767 & 105.7322 & 130.8676 & 92.28544 \\
\hline AIC & 105.997 & 196.4153 & 215.4644 & 263.7352 & 188.5709 \\
\hline AICC & 106.3662 & 196.5972 & 215.6462 & 263.7949 & 188.7527 \\
\hline BIC & 112.6993 & 200.8836 & 219.9326 & 265.9693 & 193.0391 \\
\hline HQIC & 108.656 & 198.188 & 217.2371 & 264.6216 & 190.3436 \\
\hline $\begin{array}{l}\text { KS-Distance } \\
\text { (D) }\end{array}$ & 0.057772 & 0.355 & 0.36154 & 0.44828 & 0.31119 \\
\hline $\begin{array}{l}\text { Likelihood } \\
\text { ratio statistic }\end{array}$ & 92.418 & & & \\
\hline $\begin{array}{l}\text { ML Estimates } \\
\hat{c}=21.760292 \\
(5.169830)\end{array}$ & $\begin{array}{l}\hat{\alpha}=0.001000 \\
\hat{\theta}=0.98255426 \\
(0.05831841) \\
(958.215785) \\
\hat{\theta}=9.793707 \\
(1.716879)\end{array}$ & $\begin{array}{l}\hat{\theta}=0.8156695 \\
(0.06359975)\end{array}$ & $(0.2951056)$ & $\begin{array}{l}\hat{\theta}=1.22351031 \\
(0.06779732)\end{array}$ \\
\hline
\end{tabular}

Table 9: ML estimates, -logL, AIC, AICC, BIC, HQIC, KS-distance, for fitted WLQXD and other mentioned models for data set 2.

\begin{tabular}{|c|c|c|c|c|c|}
\hline Distribution & WLQXD & LQXD & QLD & ED & QAD \\
\hline$-\log L$ & 23.75059 & 73.63075 & 66.34654 & 88.83032 & 54.55285 \\
\hline AIC & 53.50119 & 151.2615 & 136.6931 & 179.6606 & 113.1057 \\
\hline AICC & 53.90797 & 151.4615 & 136.8931 & 179.7262 & 113.3057 \\
\hline BIC & 59.93059 & 155.5478 & 140.9793 & 181.8038 & 117.392 \\
\hline HQIC & 56.0299 & 152.9473 & 138.3789 & 180.5035 & 114.7915 \\
\hline KS-Distance (D) & 0.2156 & 0.30991 & 0.34707 & 0.418 & 0.30549 \\
\hline $\begin{array}{l}\text { Likelihood ratio } \\
\text { statistic }\end{array}$ & & 99.760 & & & \\
\hline ML Estimates & $\begin{array}{l}\hat{c}=14.829858 \\
\hat{\alpha}=0.001000 \\
\hat{\theta}=11.732504 \\
(1.464477)\end{array}$ & $\begin{array}{l}\hat{\alpha}=0.001000 \\
\hat{\theta}=1.4647349 \\
(0.1004913)\end{array}$ & $\begin{array}{l}\hat{\alpha}=0.001000 \\
\hat{\theta}=1.3269391 \\
(0.1075059)\end{array}$ & $\begin{array}{c}\hat{\theta}=1.506826 \\
(0.189842)\end{array}$ & $\begin{array}{l}\hat{\alpha}=0.001000 \\
\hat{\theta}=1.9900949 \\
(0.1132172)\end{array}$ \\
\hline
\end{tabular}


Different criteria's of goodness of fit like AIC, BIC, AICC, HQIC and K-S distance have been computed by using $\mathrm{R}$ software for both the data sets and it has been observed from Tables 8 and 9 that proposed model possesses lesser AIC, BIC, AICC, HQIC \& K-S distance values as compared to Lindley Quasi Xgamma distribution, Quasi Lindley distribution, Exponential distribution and Quasi Akash distribution for both the data sets. Hence proposed model provides a good fit to both the mentioned data sets.

$$
\begin{array}{ll}
\mathrm{AIC}=2 \mathrm{k}-2 \log L & \mathrm{AICC}=\mathrm{AIC}+\frac{2 k(k+1)}{n-k-1} \\
\mathrm{BIC}=\mathrm{k} \log n-2 \log L & \mathrm{HQIC}=2 \mathrm{k} \log (\log (\mathrm{n}))-2 \log L
\end{array}
$$

Where $\mathrm{k}=$ number of parameters in model

$\mathrm{n}=$ size of the sample (number of observations in data set)

$\log \mathrm{L}=$ value of likelihood function of model

Likelihood ratio statistic is used for testing the significance of weight parameter in both the data sets. For testing $H_{0}: c=0$ versus $H_{1}: c \neq 0$ the LR statistic for testing $H_{0}$ is $\omega_{1}=2\left\{L(\hat{\Theta})-L\left(\hat{\Theta}_{0}\right)\right\}=92.418$ for data set 1 as in Table 8, $\omega_{2}=2\left\{L(\hat{\Theta})-L\left(\hat{\Theta}_{0}\right)\right\}=99.760$ for data set 2 as in Table 9, where $\hat{\Theta}$ and $\hat{\Theta}_{0}$ are MLEs under $H_{1}$ and $H_{0}$.LR statistic $\omega \sim\left(\chi_{(1)}{ }^{2}(\alpha=0.05)=3.841\right)$ as $n \rightarrow \infty$, where $1=$ degrees of freedom is the difference in dimensionality. From Table $8 \omega_{1}=92.418>3.841 \&$ from Table $9 \omega_{2}=99.760>3.841$ at $5 \%$ level of significance for both the data sets, so we reject $H_{0}$ and conclude that weight parameter $c$ plays statistically a significant role. 
Histogram of data

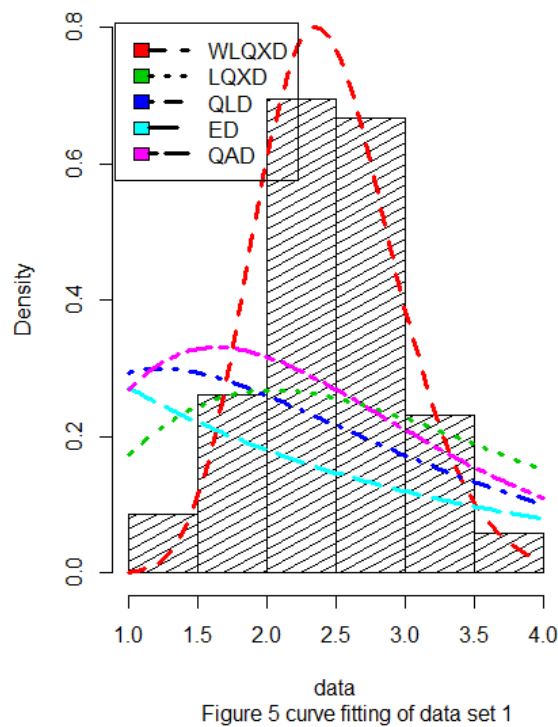

Histogram of data

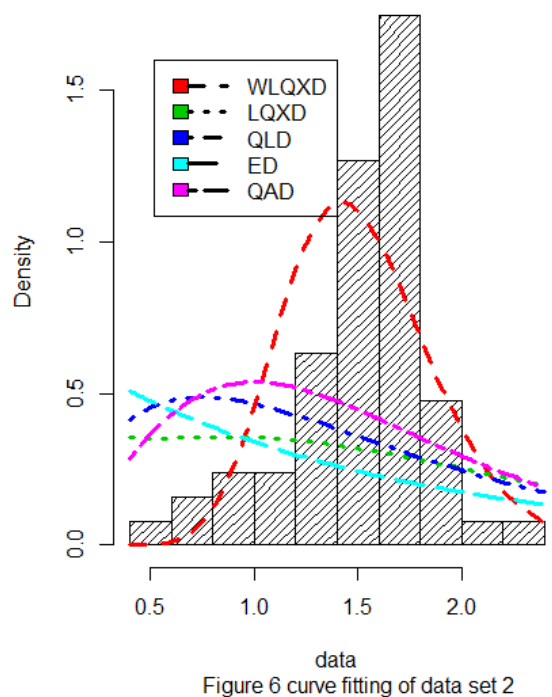

\section{CONCLUSION}

Generalized version of Lindley-Quasi Xgamma distribution by using weighting technique has been proposed in this paper. We obtained the important statistical properties like moments, reliability, moment generating function, order statistics, Bonferroni and Gini indices of formulated model. Expression for Renyi entropy has been derived. For obtaining the estimates of unknown parameters maximum likelihood estimation method is used. For testing the suitability of ML estimates simulation study has been carried which showed that ML estimation method performs well for proposed model. For testing the goodness of fit of proposed model and for investigating the application of proposed model in real life we fitted our proposed model and its related models to two real life data sets and computed log-likelihood values, AIC, AICC, HQIC, BIC and Kolmogorov statistic (D). We observed that our model possesses lesser values of AIC, BIC, AICC, HQIC and D values. Hence our model finds greater applicability in modeling survival times. From generated data as well as from real life data significant role of weight parameter has been observed. 


\section{REFERENCES}

[1] W. G. Warren. "Statistical Distributions in Forestry and Forest Products Research", A Modern Course on Statistical Distributions in Scientific Work, 369-384.

[2] A. Hassan, S. A. Dar and B. A. Para. "A New Generalization of Ishita Distribution: Properties and Applications", Journal of Applied Probability and Statistics, 13(1), 2019.

[3] A. Hassan, S. A. Wani and B. A. Para. "On three Parameter Weighted Quasi Lindley Distribution: Properties and Applications", International Journal of Scientific Research in Mathematical and Statistical Sciences, 5(5), 210-224, 2018.

[4] K. K. Das and T. B. Roy. "Applicability of Length Biased Weighted Generalized Rayleigh Distribution”, Advances in Applied Science Research, 2(4), 320-327, 2011.

[5] G. P. Patil and C. R. Rao. "Weighted Distributions and Size Biased Sampling with Applications to Wild Life Populations and Human Families”, Biometric, 34, 179-189, 1978.

[6] A. Hassan, S. A. Wani and S. Shafi. "Poisson Pranav Distribution and its Applications", Pak. J. Statist, 36(1), 57-72, 2020.

[7] S. Rezaeia, S. Nadarajah and N. Tahghighnia. "A New three Parameter Lifetime Distribution", J Theor App Statist, 47(4), 835-860, 2013.

[8] A. Hassan, S. A. Wani, B. A. Sheikh and N. Akhtar. "A New Generalized Quasi Xgamma Distribution Applicable to Survival Times", Journal of Xi'an University of Architecture and Technology, XII(IV), 3720-3736, 2020.

[9] A. Hassan, S. A. Wani, S. Shafi and B. A. Sheikh. "Lindley-Quasi Xgamma Distribution: Properties and Applications", Pak. J. Statist, 36(1), 73-89, 2020.

[10] M. G. Badar and A. M. Priest. "Statistical aspects of fiber and bundle strength in hybrid composites", Progress in Science and Engineering Composites, Tokyo, ICCM-IV, 11291136, 1982.

[11] R. L. Smith and J. C. Naylor. "A comparison of maximum likelihood and Bayesian estimators for three-parameter weibull distribution”, Applied Stat., 36, 358-369, 1987.

[12] S. Mudasir and S. P. Ahmad. "Structural Properties of Length Biased Nakagami Distribution", International journal of Modern Mathematical Sciences, 13(3), 217-227, 2015.

[13] R Core Team. R version 3.5.3, "A language and environment for statistical computing. R Foundation for Statistical Computing”, Vienna, Austria, 2019, URL https://www.Rproject.org/. 
Sameer Ahmad Wani

Department of Statistics University of Kashmir,

Srinagar 190006, Jammu \& Kashmir, India

email: wanisameer199@gmail.com (corresponding author)

Shaista Shafi

Department of Statistics University of Kashmir, Srinagar 190006, Jammu \& Kashmir, India email: sannabhat02@gmail.com

corresponding author: Sameer Ahmad Wani 